\title{
VARIATION OF PARAMETERS FORMULA FOR THE EQUATION OF COOKE AND WIENER
}

\author{
K. N. JAYASREE AND S. G. DEO \\ (Communicated by Kenneth R. Meyer)
}

\begin{abstract}
A variation of parameters and Gronwall type integral inequality are proved for a differential equation involving piecewise alternately retarded and advanced argument.
\end{abstract}

\section{INTRODUCTION}

In [3] Cooke and Wiener studied a new differential equation alternately of retarded and advanced type. They have shown that all equations with piecewise constant delays have characteristics similar to the equations studied in [2]. These equations are closely related to impulse and loaded equations and, especially, to difference equations of a discrete argument. The equations are similar in structure to those found in certain "sequential-continuous" models of disease dynamics [1].

The method of variation of parameters and Gronwall type integral inequalities are some of the most important techniques in the study of the qualitative properties of linear and nonlinear differential equations [5]. In particular, the study of perturbation theory heavily depends on these methods.

In this paper we establish variation of parameters formula for equation (1.3) and also prove an integral inequality. Consider for $t \geq 0$ the following equations

$$
\begin{aligned}
x^{\prime}(t) & =a(t) x(t), \\
y^{\prime}(t) & =a(t) y(t)+c(t) y(2[(t+1) / 2], \\
z^{\prime}(t) & =a(t) z(t)+c(t) z(2[(t+1) / 2])+f(t),
\end{aligned}
$$

with initial conditions:

$$
x(0)=y(0)=z(0)=c_{0},
$$

Received by the editors August 22, 1989 and, in revised form, February 25, 1990.

1980 Mathematics Subject Classification (1985 Revision). Primary 34K05, 34K15; Secondary $34 \mathrm{~K} 20$.

Key words and phrases. Variation of parameters formula, Gronwall integral inequality, alternately retarded and advanced argument.

The first author is supported by CSIR, India grant. 
where $a, c, f$ are real-valued continuous functions of $t$ defined on $[0, \infty)$. Here the symbol [ ] represents the greatest integer function. Observe that the argument deviation $t-2[(t+1) / 2]$ is negative for $2 n-1 \leq t<2 n$ and positive for $2 n<t<2 n+1$ ( $n$ is an integer). Therefore, (1.2) and (1.3) are of advanced type on $[2 n-1,2 n)$ and of retarded type on $(2 n, 2 n+1)$.

It has been established in [3] that if

$$
\int_{2 n-1}^{2 n} u^{-1}(t) c(t) d t \neq u^{-1}(2 n), \quad n=1,2,3, \ldots,
$$

where $u^{-1}$ is the reciprocal of $u$ and $u(t)=\exp \left(\int_{0}^{t} a(s) d s\right)$, then problem (1.2), (1.4) has a unique solution. Also, if $a, c$, are constant functions in (1.2), then the solution is given by

$$
y(t)=\lambda(t-2[(t+1) / 2])\left(\frac{\lambda(1)}{\lambda(-1)}\right)^{[(t+1) / 2]} c_{0} \quad \text { if } \lambda(-1) \neq 0
$$

and

$$
\lambda(t)=\exp (a t)\left(1+a^{-1} c\right)-a^{-1} c .
$$

Let $\phi$ denote the fundamental solution of (1.1) such that $\phi(0)=1$.

Next we define the fundamental solution of (1.2).

Definition. A solution $\psi(t)$ of $(1.2)$ is said to be a fundamental solution if it satisfies (1.2) with the initial condition $\psi(0)=1$.

\section{VARIATION OF PARAMETERS METHOD}

Let $x(t), y(t), z(t)$ be solutions of (1.1), (1.2), (1.3), respectively, satisfying the initial condition (1.4). It is natural to expect that the solutions $x, y$, and $z$ are related to each other. This relationship is established below through the method of variation of parameters formula. We use below the notation $\psi(t, k)=\psi(t) \psi^{-1}(k), k=1,2,3, \ldots$

Theorem 1. The unique solution of (1.3), (1.4) is given by

$$
\begin{aligned}
z(t)= & y(t)+\sum_{k=0}^{[(t+1) / 2]-1} \lambda^{-1}(1) \int_{2 k}^{2 k+1} \psi(t, 2 k) \phi(2 k+1, s) f(s) d s \\
& -\sum_{k=1}^{[(t+1) / 2]} \lambda^{-1}(-1) \int_{2 k}^{2 k-1} \psi(t, 2 k) \phi(2 k-1, s) f(s) d s \\
& +\int_{2[(t+1) / 2]}^{t} \phi(t, s) f(s) d s,
\end{aligned}
$$

where $\phi$ and $\psi$ are fundamental solutions of (1.1) and (1.2), respectively, and $y(t)$ is the solution of (1.2), (1.4).

Proof. Let $\tilde{z}(t)$ represent the integral terms on the right-hand side of (2.1). It suffices to prove that $\widetilde{z}(t)$ is a solution of $(1.3)$ and then use the superposition principle. 
Differentiate $\widetilde{z}(t)$ and use (1.1) and (1.2), to obtain

$$
\begin{aligned}
\widetilde{z}^{\prime}(t)= & \sum_{k=0}^{[(t+1) / 2]-1} \int_{2 k}^{2 k+1}(a(t) \psi(t, 2 k)+c(t) \psi(2[(t+1) / 2], 2 k)) \phi(2 k+1, s) f(s) d s \\
& -\sum_{k=1}^{[(t+1) / 2]} \lambda^{-1}(-1) \int_{2 k}^{2 k-1}(a(t) \psi(t, 2 k)+c(t) \psi(2[(t+1) / 2], 2 k)) \phi(2 k-1, s) f(s) d s \\
& +\int_{2[(t+1) / 2]}^{t} a(t) \phi(t, s) f(s) d s+f(t) \\
= & a(t) \tilde{z}(t)+c(t) \tilde{z}(2[(t+1) / 2])+f(t) .
\end{aligned}
$$

The proof is complete.

For the purpose of simplicity, we prove the next theorem which verifies the relation (2.1) for equation (1.3) with constant coefficients. The result can be generalized to equations of the type (1.3) with minor modifications.

Theorem 2. The unique solution $z(t)$ of (1.3), (1.4) with constant functions $a(t)=a, c(t)=c$, on $[0, \infty)$ is given by the relation $(2.1)$ where $\phi(t)=\exp (a t)$ and $\psi(t)$ is given by (1.5) with $c_{0}=1$ and $y(t)$ is the corresponding solution of (1.2), (1.4).

Proof. Assume that $y_{n}(t)$ and $z_{n}(t)$ are solutions of (1.2) and (1.3) in the interval $[2 n-1,2 n+1)$, respectively. Further, let $z_{n}(2 n)=d_{2 n}$, for $n=$ $0,1,2, \ldots$.

It is easy to verify that

$$
z_{n}(t)=d_{2 n} \lambda(t-2 n)+\int_{2 n}^{t} \exp \{a(t-s)\} f(s) d s,
$$

where $\lambda$ is given in (1.6). From (2.2), we obtain

$$
z_{n}(2 n-1)=d_{2 n-1}=d_{2 n} \lambda(-1)+\int_{2 n}^{2 n-1} \exp \{a(2 n-1-s)\} f(s) d s
$$

and in the limit

$$
z_{n}(2 n+1)=d_{2 n+1}=d_{2 n} \lambda(1)+\int_{2 n}^{2 n+1} \exp \{a(2 n+1-s)\} f(s) d s .
$$

Eliminating $d_{2 n}$, we get

$$
\begin{aligned}
d_{2 n+1}= & \frac{\lambda(1)}{\lambda(-1)}\left\{d_{2 n-1}-\int_{2 n}^{2 n-1} \exp \{a(2 n-1-s)\} f(s) d s\right\} \\
& +\int_{2 n}^{2 n+1} \exp \{a(2 n+1-s)\} f(s) d s .
\end{aligned}
$$


Applying (2.4) repeatedly for $d_{2 n-1}, d_{2 n-3}, \ldots, d_{3}$ yields

$$
\begin{aligned}
d_{2 n+1}= & \left(\frac{\lambda(1)}{\lambda(-1)}\right)^{n} d_{1}+\sum_{k=1}^{n}\left(\frac{\lambda(1)}{\lambda(-1)}\right)^{n-k} \\
& \times\left(\frac{-\lambda(1)}{\lambda(-1)} \int_{2 k}^{2 k-1} \exp \{a(2 k-1-s)\} f(s) d s\right. \\
& \left.\quad+\int_{2 k}^{2 k+1} \exp \{a(2 k+1-s)\} f(s) d s\right)
\end{aligned}
$$

for $n=0,1,2,3, \ldots$. From (2.2), one gets

$$
d_{1}=z_{0}(1)=\lambda(1) d_{0}+\int_{0}^{1} \exp \{a(1-s)\} f(s) d s .
$$

Use (2.5) to obtain the value of $d_{2 n-1}$ and then use (2.3) and (2.6) to find $d_{2 n}$. Substitute $d_{2 n}$ in (2.2) and use the fact $d_{0}=c_{0}$ to obtain finally

$$
\begin{aligned}
z_{n}(t)= & y_{n}(t)+\sum_{k=0}^{n-1} \lambda^{-1}(1) \int_{2 k}^{2 k+1} \lambda(t-2 n)\left(\frac{\lambda(1)}{\lambda(-1)}\right)^{n-k} \exp \{a(2 k+1-s)\} f(s) d s \\
& -\sum_{k=1}^{n} \lambda^{-1}(-1) \int_{2 k}^{2 k-1} \lambda(t-2 n)\left(\frac{\lambda(1)}{\lambda(-1)}\right)^{n-k} \exp \{a(2 k-1-s)\} f(s) d s \\
& +\int_{2 n}^{t} \exp \{a(t-s)\} f(s) d s ; \quad 2 n-1 \leq t<2 n+1 .
\end{aligned}
$$

If we take $n=[(t+1) / 2]$, then (2.7) is true for any $t$ and hence, write $z_{n}(t)=$ $z(t), y_{n}(t)=y(t)$, for $t \geq 0$. Observe that

$$
\lambda(t-2 n)\left(\frac{\lambda(1)}{\lambda(-1)}\right)^{n-k}=\psi(t, 2 k)
$$

and hence, we get (2.7) in the form (2.1).

\section{GRONWALL TYPE INTEGRAL INEQUALITY}

Integral inequalities play a useful role in the study of the qualitative behavior of solutions of linear and nonlinear differential equations. We extend the wellknown Gronwall inequality further in the following theorem.

Theorem 3. Let $c_{0}, a, c$ be nonnegative constants and $u \in C\left[[0, \infty), R^{+}\right]$. If the inequality

$$
u(t) \leq c_{0}+\int_{0}^{t}(a u(s)+c u(2[(s+1) / 2])) d s, \quad t \in[0, \infty),
$$

holds and $\lambda(-1) \neq 0$ then for $t \geq 0$

$$
u(t) \leq c_{0} \lambda(t-2[(t+1) / 2])\left(\frac{\lambda(1)}{\lambda(-1)}\right)^{[(t+1) / 2]},
$$

where $\lambda$ is defined in (1.6). 
Proof. From (3.1) we have, in $[2 n, 2 n+1)$,

$$
u(t) \leq u(2 n)+\int_{2 n}^{t} a u(s) d s+\int_{2 n}^{t} c u(2 n) d s .
$$

Therefore,

$$
u(t) \leq u(2 n) \exp \left(\int_{2 n}^{t} a d s\right)+\int_{2 n}^{t} c u(2 n) \exp \left(\int_{s}^{t} a d \tau\right) d s,
$$

and hence,

$$
u(2 n+1) \leq u(2 n)\left\{\exp (a)\left(1+a^{-1} c\right)-a^{-1} c\right\} .
$$

Similarly, in the interval $[2 n-1,2 n]$, we obtain

$$
u(2 n) \leq u(2 n-1) \exp (a)+a^{-1} c u(2 n)\{\exp (a)-1\}
$$

which leads to

$$
u(2 n) \leq u(2 n-1)\left\{\exp (-a)\left(1+a^{-1} c\right)-a^{-1} c\right\}^{-1} \text {. }
$$

Applying inequalities (3.3) and (3.4) repeatedly and using (1.6) we get the desired conclusion (3.2).

Remark. Observe that the right-hand side of inequality (3.2) is in fact the solution of the related equation (1.2) when $a$ and $c$ are constant functions. In this sense, (3.2) is the best estimate. When $c=0$ in (3.1), (3.2) reduces to $u(t) \leq c_{0} \exp (a t), t \geq 0$.

Observation. Equation (1.3) can be further generalized to contain two types of delays, namely, continuous past history and piecewise constant argument. We can study the equation

$$
z^{\prime}(t)=a z(t)+b z(t-1)+c z(2[(t+1) / 2])+f(t), \quad t \geq 0,
$$

where $a, b, c$ are real constants with some suitable initial requirements.

Theorem 5.1 of $[4$, p. 19] provides a method of constructing the fundamental solution $\phi$ of the equation $x^{\prime}(t)=a x(t)+b x(t-1)$.

Also, using the relation $(6.1)$ of $[4$, p. 21$]$ we can construct the fundamental solution $\psi$. Once the functions $\phi$ and $\psi$ are available, the variation of parameters formula given in Theorem 1 can be extended for equation (3.5). The same procedure is applicable with respect to the functional differential equations of the form

$$
z^{\prime}(t)=a z(t)+b L(z(t+\theta))+c z(2[(t+1) / 2])+f(t), \quad t \geq 0,
$$

where $L$ is a linear operator defined in [4, p. 142].

\section{ACKNOWLEDGMENT}

The authors sincerely thank the referee for comments and useful suggestions to improve the paper. 


\section{REFERENCES}

1. S. Busenberg and K. L. Cooke, Models of vertically transmitted diseases with sequentialcontinuous dynamics, Nonlinear Phenomena in Mathematical Sciences (V. Lakshmikantham, ed.), Academic Press, New York, 1982, pp. 179-187.

2. K. L. Cooke and J. Wiener, Retarded differential equations with piecewise constant delays, J. Math. Anal. Appl. 99 (1984), 265-297.

3. $\ldots$ An equation alternately of retarded and advanced type, Proc. Amer. Math. Soc. 99 (1987), 726-732.

4. J. K. Hale, Theory of functional differential equations, Applied Mathematical Sciences, vol. 3, Springer-Verlag, New York, 1976.

5. V. Lakshmikantham and S. Leela, Differential and integral inequalities, vol. 1, Academic Press, New York, 1969.

Department of Mathematics, Goa University, P. O. Santacruz, Goa 403005, India . 